\title{
Silencing of HMGA2 reverses retardance of cell differentiation in human myeloid leukaemia
}

\author{
Li Tan ${ }^{\star}, 1,2$, Hongfa $X u^{1}$, Guoshu Chen ${ }^{3}$, Xiaoping $\mathrm{Wei}^{4}$, Baodan $\mathrm{Yu}^{4}$, Jingmei $\mathrm{Ye}^{1}$, Lihua $\mathrm{Xu}{ }^{1,2}$ and Huo Tan ${ }^{1}$ \\ ${ }^{1}$ Department of Hematology, The First Affiliated Hospital of Guangzhou Medical University, Guangzhou 510230, China; \\ ${ }^{2}$ Department of Urology, Minimally Invasive Surgery Center, The First Affiliated Hospital of Guangzhou Medical University, \\ Guangdong Key Laboratory of Urology, Guangzhou 510230, China; ${ }^{3}$ Department of Hematology, Huizhou Municipal Central \\ Hospital, Huizhou 516001, China and ${ }^{4}$ Clinical Laboratory, The First Affiliated Hospital of Guangzhou Medical University, \\ Guangzhou 510120, China
}

Background: High-mobility group AT-hook 2 (HMGA2) may serve as an architectural transcription factor, and it can regulate a range of normal biological processes including proliferation and differentiation. Upregulation of HMGA2 expression is correlated to the undifferentiated phenotype of immature leukaemic cells. However, the underlying mechanism of HMGA2-dependent myeloid differentiation blockage in leukaemia is unknown.

Methods: To reveal the role and mechanism of HMGA2 in differentiation arrest of myeloid leukaemia cells, the quantitative expression of HMGA2 and homeobox A9 (HOXA9) was analysed by real-time PCR (qRT-PCR). The regulatory function of HMGA2 in blockage of differentiation in human myeloid leukaemia was investigated through in vitro assays (XTT assay, May-GrünwaldGiemsa, flow cytometry analysis and western blot).

Results: We found that the expression of HMGA2 and HOXA9 was reduced during the process of granulo-monocytic maturation of acute myeloid leukaemia (AML) cells, knockdown of HMGA2 promotes terminal (granulocytic and monocytic) differentiation of myeloid leukaemia primary blasts and cell lines, and HOXA9 was significantly downregulated in leukaemic cells with knockdown of HMGA2. Downregulation of HOXA9 in myeloid leukaemia cells led to increased differentiation capacity in vitro.

Conclusions: Our data suggest that increased expression of HMGA2 represents a possible new mechanism of myeloid differentiation blockage of leukaemia. Aberrant expression of HMGA2 may enhance HOXA9-dependent leukaemogenesis and myeloid leukaemia phenotype. Disturbance of the HMGA2-HOXA9 pathway is probably a therapeutic strategy in myeloid leukaemia.

The most common type of leukaemia is AML in adults, which is about one-third of newly diagnosed patients. The characteristics of myeloid leukaemia are arrested differentiation of clonal precursor myeloid cells (McCulloch, 1983). Myeloid leukaemia is also a heterogeneous neoplasia with arrested leukaemic blasts, and these cells accumulate in various periods of granulocytic and monocytic differentiation (Bennett et al, 1985), but the targets of differentiation in myeloid leukaemia remain largely unknown. Current treatment of AML depends on remission induction with Ara-C and anthracyclines (e.g., idarubicin and daunorubicin), but they have distinct morbidity and mortality, especially in elderly patients (Stone, 2009), although the majority of new AML patients can achieve remission. However, relapse is universal and long-term survival is usually low. In contrast with the poor prognosis for

*Correspondence: Professor L Tan; E-mail: tanya_tanli@163.com

Received 13 April 2017; revised 7 October 2017; accepted 12 October 2018; published online 2 January 2018

(C) 2018 Cancer Research UK. All rights reserved 0007-0920/18 
most of the myeloid leukaemia patients, acute promyelocytic leukaemia (APL) can be treated by inducing differentiation using all-trans retinoic acid (ATRA), which introduced a successful example of cell differentiation treatment for AML (Wang and Chen, 2008). Unfortunately, inherent resistance to ATRA-inducing differentiation was shown in the other AML subtypes. Furthermore, resistance to ATRA may occur in many APL patients and after treatment with ATRA, APL always relapses. Thus, it is necessary to develop new agents for the therapy of myeloid leukaemia, especially the ones that utilise differentiation pathways.

Recent studies suggested that HMGA2 is associated with different tumours, including leukaemia (Tan et al, 2016). HMGA2 protein is an architectural transcription factor including an acidic carboxy-terminal tail and three DNA-binding domains, these domains are considered to be AT hooks. Via protein-protein or protein-DNA interactions, HMGA2 influences various normal biological processes including differentiation, proliferation or death (Cleynen and Van de Ven, 2008). The human HMGA2 gene is normally expressed only during embryonic/foetal development but in none of the adult tissues tested so far (Cleynen and Van de Ven, 2008). Misexpression of alternative HMGA2 transcripts was recently described in leukaemia samples; HMGA2 is widely involved in haematological malignancies and plays an important role in myeloid malignancies (Odero et al, 2005). However, little information is as yet available on their relationship to events related to differentiation of leukaemic cells, and the mechanisms of HMGA2 involved in myeloid differentiation blockage of leukaemia were still worth further studying. Some results indicated that homeobox A9 (HOXA9) gene possesses medullary system specificity; HOXA9 is overexpressed in $70 \%$ of patients with AML and implicated in the immature leukaemia cells, likely reflecting that the leukaemic blasts are halted at a common phase of differentiation stagnation (Golub et al, 1999; Thorsteinsdottir et al, 2001). HOXA9 might take effect in the pathogenesis of AML (Thorsteinsdottir et al, 2002; Faber et al, 2009). Some results show that HMGA2 can regulate HOXA9 during breast cancer growth and metastasis (Sun et al, 2013), but the connection between them during myeloid differentiation blockage of leukaemia has not yet been investigated.

Therefore, in the current work, we have explored the role of HMGA2 in differentiation arrest of myeloid leukaemia cells. As both HMGA2 and HOXA9 play important roles in myeloid leukaemogenesis, we hypothesise that HOXA9 is among the upregulated genes of HMGA2 in undifferentiated status of myeloid leukaemia cells. To test this, we have utilised stable HMGA2 knockdown strategies and tested HOXA9 expression with or without chemical treatment and HMGA2 knockdown. So, the mechanisms underlying HMGA2 regulation in modulating myeloid leukaemia cell differentiation were also explored.

\section{MATERIALS AND METHODS}

Primary AML specimens. Our study was approved by the Ethics Committee of the First Affiliated Hospital of Guangzhou Medical University. Ten adult patients in the 16 and 79 age range are analysed in the current study, with M7 $(n=1)$, M5 $(n=1)$, M4 $(n=1)$, M3 $(n=2)$, M2 $(n=2)$, M1 $(n=1)$ or M0 $(n=2)$ leukaemia (FAB classification). Primary leukaemia cell specimens were obtained from preliminarily diagnosed AML patients who have not received any treatment before specimens are collected. Ficoll-Hypaque density gradient centrifugation was used to purify mononuclear cells in blood. As needed, samples were thawed and used immediately. We completed CD34 + cell selection by using the Miltenyi (Auburn, CA, USA) immunoaffinity device (varioMACS) in the light of the manufacturer's instructions. We cultured CD34+ primary cells in IMDM medium (StemCell Technologies Inc) supplemented with streptomycin, penicillin, $10 \%$ foetal bovine serum, $50 \mathrm{ng} \mathrm{ml}^{-1} \mathrm{FLT}-3$ ligand, $50 \mathrm{ng} \mathrm{ml}^{-1}$ stem cell factor, $50 \mathrm{ng} \mathrm{ml}^{-1} \mathrm{GM}-\mathrm{CSF}$ and $10 \mathrm{ng} \mathrm{ml}^{-1}$ IL-3. Transient transfections were performed on $10^{6}$ cells. For in vitro studies, cells were cultured in serum-free medium for overnight before the addition of lentivirus. The next day, cells were transduced with lentiviral supernatants at MOI of 300, and then, we centrifuged $(1800 \mathrm{~g})$ the transduction mixture for $4 \mathrm{~h}$ at $32^{\circ} \mathrm{C}$ $35^{\circ} \mathrm{C}$ as described before (Gao et al, 2001). We repeated the transduction procedure in the following day, and then changed the medium, and cultured the cells for additional $48 \mathrm{~h}$ to allow gene expression. Differentiation was evaluated after $6 \mathrm{~d}$ of differentiation led by GM-CSF and IL-3 treatment and transient transfection (at $48 \mathrm{~h}$ ) of the shControl or shHMGA2. All the patients were informed with the informed consents obtained in the present study.

RNA extraction and real-time quantitative reverse transcription PCR. We enriched mononuclear cells from specimens of AML patients at preliminary diagnosis and healthy donors by FicollHypaque gradient and isolated the total RNA by the TRIzol reagent (Invitrogen, Carlsbad, CA, USA) according to the manufacturer's instructions. Two micrograms of total RNA, RNase inhibitor, MMLV reverse transcriptase (MBI Fermentas, Hanover, MD, USA), dNTPs and random primers were used to synthesise cDNA. In 20- $\mu \mathrm{l}$ reaction volume including $0.4 \mu \mathrm{M}$ ROX Reference Dye1 and $10 \mu \mathrm{M}$ AceQTMqPCR SYBR Green Master Mix (Vazyme Biotech Co, USA), qRT-PCR assay was carried out for each specimen in duplicate. HOXA9 was amplified with the primers described previously (Zhao et al, 2015). The primers of amplifying endogenous control gene GAPDH and HMGA2 gene, reaction conditions and interpretation method of result were the same as those described previously (Tan et al, 2016).

Flow cytometry. For CD11b, CD13, CD14 and CD15 determinations, we washed cells with FACS buffer (PBS supplemented with $0.05 \%$ sodium azide and $5 \% \mathrm{FBS}$ ), and then, we stained cells by using PE-conjugated anti-CD13, CD14 and CD15 and fluoroscein isothiocyanate (FITC)-conjugated anti-CD11b monoclonal antibodies (BD Biosciences, Oxford, UK) for $30 \mathrm{~min}$ on ice. We washed away the redundant antibody by resuspending the cells in $1 \mathrm{ml}$ of $5 \%$ FCS/PBS and cells were centrifugated at $1000 \mathrm{~g}$ for $6 \mathrm{~min}$. We ultimately resuspended cells in $400 \mu \mathrm{l}$ of $5 \%$ FCS/PBS for FACS analysis. We can exclude the dead cells and debris from analysis by gating on forward and side scatter parameters.

Cell lines. The NB4 (human acute promyelocytic leukaemia) and HL-60 (human acute myelogenous leukaemia) were purchased from ATCC (American Type Culture Collection, Manassas, VA, USA), and the K562 (human chronic myelogenous leukaemia) was supplied by Sun Yat-sen University Cancer Center. The NB4, K562 and HL-60 were cultured in RPMI-1640 (Invitrogen, Carlsbad, $\mathrm{CA})$. All cells were grown in their specific medium supplemented with 100 units per $\mathrm{ml}$ penicillin, and $100 \mu \mathrm{g} \mathrm{ml}^{-1}$ streptomycin (Life Technologies, Gaithersburg, MD, USA) and $10 \%$ foetal calf serum (Invitrogen, Carlsbad, CA, USA), at $37^{\circ} \mathrm{C}, 5 \% \mathrm{CO}_{2}$ in a humidified incubator.

Lentivirus production. Lentivirus expressing HMGA2 or different shRNA oligos was purchased as described previously (Tan et al, 2016). Shed virus was collected $48 \mathrm{~h}$ after transfection and used for transduction. Stable knockdown clones were obtained under puromycin $\left(0.5-5 \mu \mathrm{g} \mathrm{ml}^{-1}\right)$ selection for 7 days.

Cell proliferation assays. We semiquantitatively monitored cell proliferation by XTT assay (Roche Diagnostics GmbH, Mannheim, Germany) described previously (Tan et al, 2016). We seeded cells treated with DMSO or ATRA in 96-well plates (1000 cell per well), 
and monitored cell growth after 3 and 6 days. We can measure the cells with active metabolism by a spectrophotometer as the absorbance at $490 \mathrm{~nm}$.

Western blot. The assay was conducted as described (Tan et al, 2016), and each assay was individually repeated for at least three times. HMGA2 antibody was bought from Cell Signaling (Boston, MA, USA). HOXA9 and $\beta$-actin antibodies were bought from Abcam (Cambridge, UK).

Analysis of myeloid differentiation. Cytospins were prepared using a cytospin centrifuge and we stained the cytospin slides with May-Grünwald-Giemsa (Sigma, St Louis, MO, USA). We examined cellular morphology using the Nikon Eclipse 90i microscope (Nikon, Melville, NY, USA). Cell morphology with May-Grunwald-Giemsa (MGG)-stained cytocentrifuge slides (Yam et al, 1971), expression of CD11b and expression of CD14 were used to evaluate monocytic differentiation as previously described. Cell morphology with MGG-stained cytocentrifuge slides (Eppinger et al, 1993), expression of CD11b and expression of CD15 were used to evaluate granulocytic differentiation as previously described. We evaluated granulocytic differentiation in K562 cells by cell morphology with MGG-stained cytocentrifuge slides and expression of CD13 as previously described (Eppinger et al, 1993). Cell morphology with MGG-stained cytocentrifuge slides and expression of CD11b (FACS) were used to evaluate $\mathrm{CD} 34+$ primary blast differentiation.

Electroporation of leukaemia cells. We performed electroporation with $600 \mathrm{nmol}$ of human HOXA9 small interference RNAs (siRNA) nucleotide sequence (siHOXA9, ONTARGETplus SMARTpool, Dharmacon) or the $600-\mathrm{nmol}$ control siRNA duplex (si-ctr, ON-TARGETplus Non-targeting pool, Dharmacon) in the transfected NB4 and HL-60 cells using the electroporation system easyject plus (Eurogentec, Seraing, Belgium) and expression of endogenous HOXA9 was examined. There is no homology between control siRNA and any human gene sequences by BLAST. In brief, we suspended $5 \times 10^{6}$ cells with $500 \mu \mathrm{l}$ of complete RPMI medium, and mixed with $600 \mathrm{nmol}$ of si-ctr or siHOXA9 in a 4$\mathrm{mm}$ electroporation cuvette, then, we incubated cells on ice for $10 \mathrm{~min}$. We transferred the cells into complete RPMI medium at a density of $1 \times 10^{6}$ cells per $\mathrm{ml}$ after electroporation with a single pulse (electrical parameters for NB4 between 300 volt, $1050 \mu \mathrm{F}$ and 400 volt, $1800 \mu \mathrm{F}, 99 \Omega$ and for HL-60 electroporation, the condition differs between 1050 and $1800 \mu \mathrm{F}, 200-450 \mathrm{~V}$ (in $50-\mathrm{V}$ steps) and $99 \Omega$ ).

Statistical analysis. Data presented here represent at least three experiments with basically similar results. The results are shown as means \pm s.d. from three independent experiments. Statistical significance was evaluated by the two-tailed Student's T-test. $P<0.05$ was considered as a significantly different value.

\section{RESULTS}

HMGA2 and HOXA9 are overexpressed in patients with AML and myeloid leukaemia cells with undifferentiation. We first tested the status of HMGA2 and HOXA9 gene by qRT-PCR in AML specimens and healthy controls. HMGA2 and HOXA9 mRNA relative expression levels were obviously upregulated in 10 new AML patients, compared with the controls $(P<0.05)$. HMGA2 and HOXA9 gene expression were obviously higher in patients with AML, compared with normal subjects (Figure $1 \mathrm{~A}$ and B). Spearmann's correlation coefficients show that HOXA9 expression was relevant with the expression of HMGA2 in 7 identical new AML patients $(r=0.883, P<0.001$; Figure $1 C)$. Western blot analysis shows that HMGA2 is also upregulated in primary AML cells and HL-60, NB4 and K562 myeloid leukaemia cell lines (Figure 1D). Here, we examined the potential role of HMGA2 in AML cell differentiation. Western blot analysis with anti-HMGA2 antibody shows that ATRA treatment significantly decreased HMGA2 expression in NB4 cells on the third day, while DMSO treatment significantly decreased HMGA2 expression in HL-60 cells on the sixth day (Figure 1E). HMGA2 gene was significantly inhibited in differentiated AML cells, compared with cells by day 0 (Figure $1 \mathrm{~F}$ ).

Chemical treatments strengthen the effect of genetic suppression of HMGA2 on cell viability in myeloid leukaemia. We hypothesised that chemical treatments would synergise with inhibition of HMGA2 in myeloid leukaemia in both its promoting differentiation and anti-viability effects. To explore the functional role of HMGA2 expression, we constructed lentivirus-HMGA2 shRNA-marked (ShHMGA2), which expresses a HMGA2 genespecific small hairpin RNA, pools of NB4 and HL-60 cells stably transfected by lentivirus-ShHMGA2 were established and the control cells were transfected by lentivirus-NC-marked (ShControl) with a scrambled hairpin. We confirmed gene knockdown of HMGA2 by RT-PCR and western blot, and the expression of HMGA2 gene could be effectively inhibited by HMGA2 shRNA transfection that is confirmed by our previous work (Tan et al, 2016). The proliferation rate of HL-60 and NB4 cells with HMGA2 low expression was obviously inhibited upon chemical treatment, as shown in Figure 2A and B, and both HMGA2 knockdown and exposure to the chemical treatments lead to more lack of cell vitality by day 6 than with any one treatment alone.

Western blot analysis confirmed HMGA2 gene knockdown in K562 cells (Figure 3A). Stable knockdown of the HMGA2 was also able to enhance the differentiation of K562 cells upon chemical treatment, and the proliferation rate of K562 cells with low expression of HMGA2 was also remarkably inhibited upon chemical treatment (Figure 3B).

Knockdown of HMGA2 significantly promotes blast differentiation in myeloid leukaemia cells. We then assessed the merged effects on differentiation by focusing on HMGA2 knockdown and AML cell lines, HL-60 and NB4. The result of morphology shows that HMGA2 knockdown in HL-60 and NB4 cells has reduced nucleus-to-cytoplasm ratio and cell size, convoluted and indentedshaped nuclei and a more mature cell morphology. To get a more effective quantitative induced differentiation, stable HMGA2 knockdown in HL-60 and NB4 cells seemingly gets a more distinct maturation of HL-60 into monocytic cells (after DMSO treatment), and NB4 into granulocytic cells (after ATRA treatment), which was further proved by the result of flow cytometry (Figure 2C), and the expression of CD14 in HL-60 cells and CD15 in NB4 cells with HMGA2 knockdown was increased (Figure 2E and F). For monocytic differentiation, the cell maturation was exhibited by morphology (Figure 2D) and increased expression of CD14 in HL60 cells with HMGA2 knockdown (Figure 2C and E), of note, it seemed that the maturation with the late stages of monocytic differentiation between the expression of CD11b and CD14 may occur. For the granulocytic differentiation, expression of CD11b was obviously affected by knockdown of HMGA2 in NB4 cells with ATRA treatment (Figure 2C). The cell maturation was confirmed by morphological analysis (Figure 2D) and increased expression of CD15 in NB4 cells with HMGA2 knockdown (Figure 2C and F), the maturation seemed to occur in late stages of granulocytic differentiation between the expression of CD11b and CD15. It was suggested that knockdown of HMGA2 significantly enhanced the differentiation towards both lineages. While cultured with $0.5 \%$ DMSO, more mature cell morphology of the K562 cells was revealed by increased cytoplasmic quantity, decreased ratio of nucleus/cytoplasm and nuclei with incisura and kidney of irregular shape. After co-incubation with $0.5 \%$ DMSO for 6 days, the number of neutrophils and later-stage cells significantly increased 


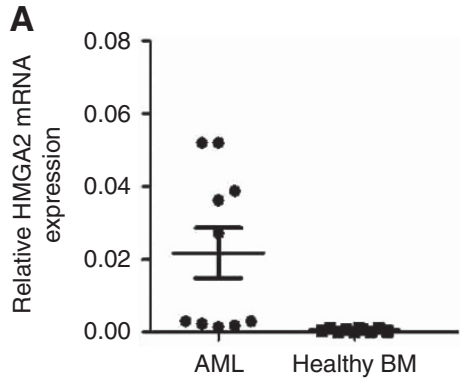

C

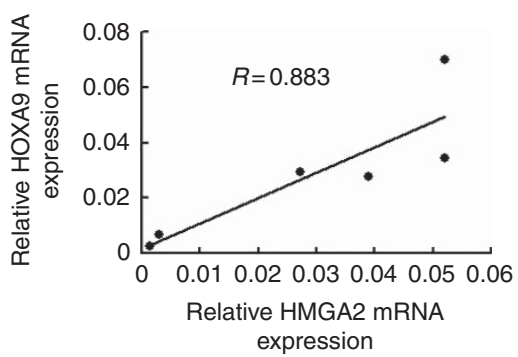

B

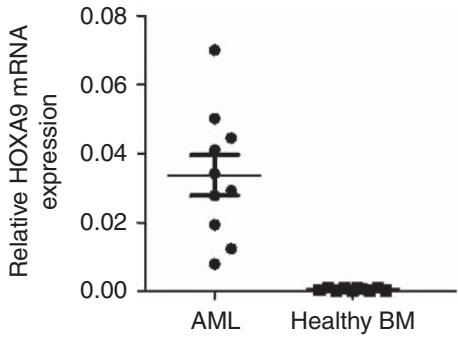

D

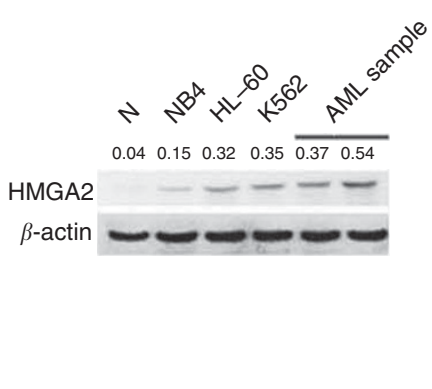

$\mathbf{E}$

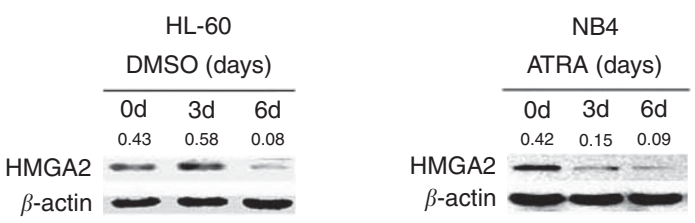

$\mathbf{F}$

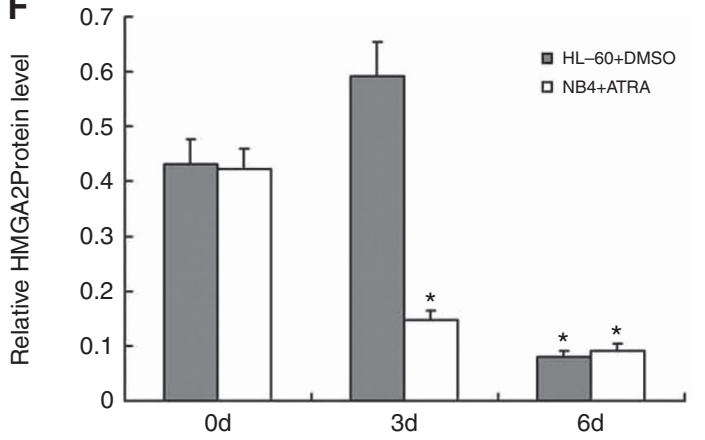

Figure 1. HMGA2 and HOXA9 mRNA relative expression levels in control and AML. (A, B) HMGA2 and HOXA9 mRNA relative expression levels of AML cells by qRT-PCR. HMGA2 and HOXA9 expression are shown after being normalised by GAPDH and calculations of $2-\Delta \Delta C \mathrm{Ct}$. Overexpression of the HMGA2 and HOXA9 in $10 \mathrm{AML}$ patients was shown compared with controls (10 BM specimens from healthy individuals; $P<0.05)$. Horizontal lines suggest mean values. (C) Analysis of qRT-PCR implemented for 7 newly diagnosed AML patients showed that HOXA9 expression was related with HMGA2 $(r=0.883, P<0.001)$. (D) Western blot analysis was carried out in NB4, HL-60, K562 cell lines and primary AML cells, and high expression of the HMGA2 in myeloid leukaemia cells was shown compared with normal control. (E) Western blot analysis was conducted using the cells exposed to either $0.5 \%$ DMSO for HL-60 or $1 \mu \mathrm{m}$ ATRA for NB4, respectively, and HMGA2 expression was analysed at 0 , 3 and 6 days after treatment. (F) $\beta$-actin was a loading control, and numbers above the lanes represent densitometric analysis of HMGA2/ $\beta$-actin. The western blots shown are from one experiment, which represents at least three independent experiments and cell lysates. Error bars denote the mean \pm s.d. of three replicates with statistical significance estimated by Student's T-test. ${ }^{\star} P<0.05$ ( $3 \mathrm{~d}$ or $6 \mathrm{~d}$ vs $0 \mathrm{~d}$ ).

in K562 cells with knockdown of HMGA2 (Figure 3C), and the expression of CD13 was also higher than that in K562 cells (Figure 3D and E). Together, these findings suggest that knockdown of HMGA2 has the granulocyte and monocytic prodifferentiation role in myeloid leukaemia cells upon chemical treatment, and knockdown of HMGA2 may induce granulomonocytic differentiation of myeloid leukaemia cells. Under proper culture conditions, differentiation along the granulocytic and/or the monocytic lineage can be induced in myeloid leukaemia cells, backing the bipotential granulo-monocytic essence in myeloid leukaemia blasts.

Knockdown of HMGA2 affects differentiation of CD34 + primary blast. We transfected $10^{6}$ cells of human $\mathrm{CD} 34+$ primary blasts with lentivirus-ShHMGA2 or lentivirus-ShControl. In consideration of the criteria used for myeloid cells in vitro, differentiation arrest that was especially obvious about morphological features was displayed by the results obtained with primary blasts. By and large, we obtained more differentiated cells upon ShHMGA2 transfection in comparison with controls (Figure $4 \mathrm{~A}$ and $\mathrm{B}$ ), which was estimated 8 days after inducing differentiation (upon GM-CSF treatment inducing both monocytic and granulocytic differentiation). For the results of FACS, there was a trend increase in the percentages of CD11b-positive cell in all experiments, as observed in NB4 and HL-60 cells (with monocytic or granulocytic differentiation), and in accordance with these findings, $\mathrm{CD} 1 \mathrm{~b}$ that increased expression in primary blasts transduced by lentivirus-ShHMGA2 from three newly diagnosed AML patients' bone marrow (Figure 4C) was also observed.
Together, these data intensely show that granulo-monocytic differentiation of AML cells may be induced by ShHMGA2.

HOXA9 expression was decreased during differentiation upon chemical treatment. Although a great deal of studies have depicted diverse targets of HMGA2, HOXA9 that may help definitely to the AML phenotype has not been identified yet. First, we checked the effect of DMSO or ATRA on the protein levels of HOXA9 for AML cells. The result of western blot showed that DMSO treatment obviously affected HOXA9 expression in HL-60 on the 6th day (Figure 5A and C), and ATRA treatment also affected HOXA9 expression in NB4 on the third day (Figure 5B and D). It was suggested that the expression of HOXA9 was decreased during the AML cell differentiation upon chemical treatment in vitro.

Knockdown of HMGA2 affects HOXA9 expression during cell differentiation of myeloid leukaemia. Next, the effect of knockdown of HMGA2 on HOXA9 protein levels in myeloid leukaemia cell differentiation was evaluated. Western blot analysis showed that HMGA2 knockdown significantly decreased HOXA9 protein levels upon DMSO or ATRA treatment, compared with control (Figure 6C, D and F). HOXA9 effectors may be differentially downregulated by knockdown of HMGA2 in myeloid leukaemia (Figure 6A, B and E).

HOXA9 is an effector in inducing the differentiation of HMGA2 knockdown in AML. The expression of HOXA9 gene could be effectively inhibited by siHOXA9 transduction. The inhibition was affirmed at the protein levels by western blot 
A



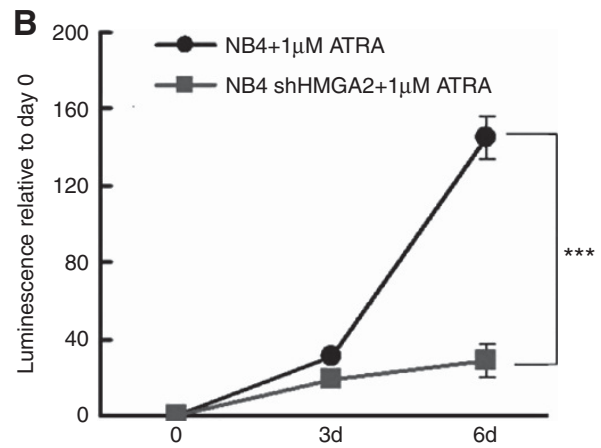

E
C
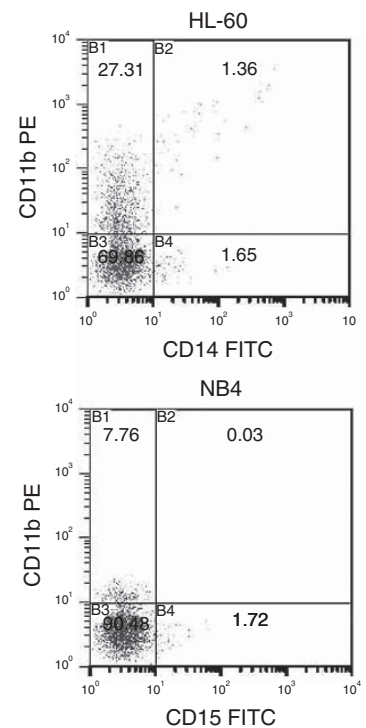

D

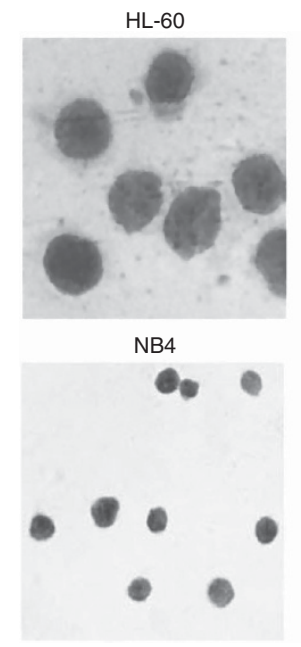

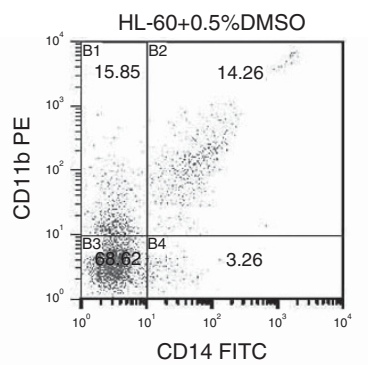

CD14 FITC

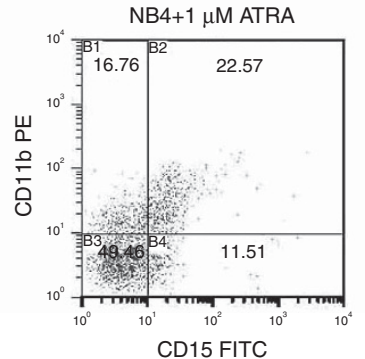

CD15 FITC

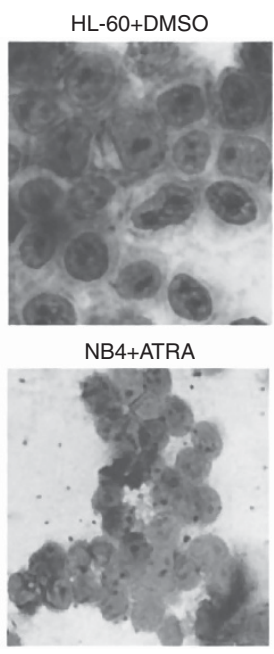

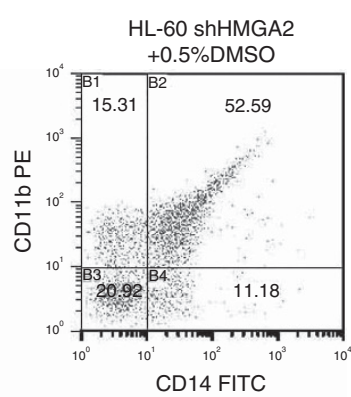

CD14 FITC

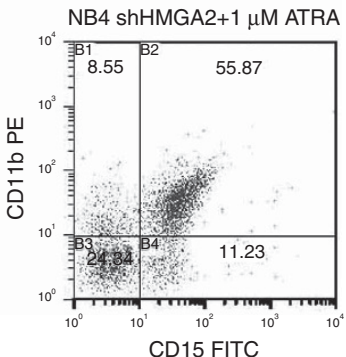

CD15 FITC

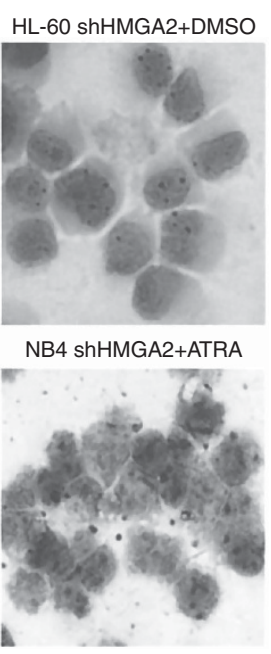

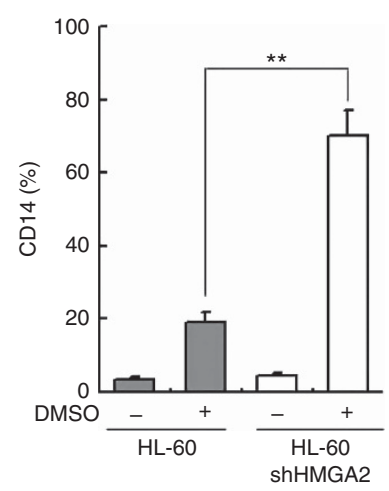

F

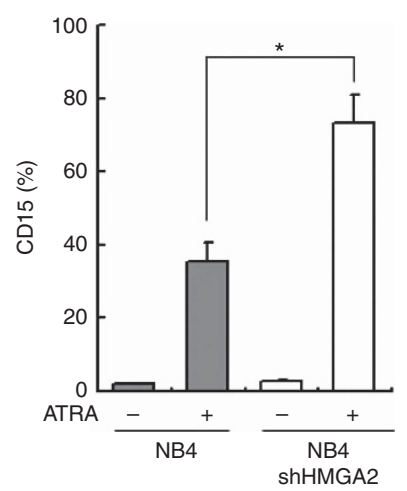

Figure 2. Chemical treatment increases the effect of genetic suppression of HMGA2 on cell viability and induces monocytic and granulocytic differentiation of AML cells. (A, B) AML cell lines with lentivirus-ShHMGA2 or lentivirus-ShControl were exposed to either $0.5 \%$ DMSO for HL-60 or $1 \mu \mathrm{m}$ ATRA for NB4 and viability was evaluated at 0, 3 and 6 days after treatment. The mean $\pm \mathrm{s}$.d. of three replicates for each compound at day 6 was shown by error bars, and three replicates have statistical significance estimated by Student's T-test. All brackets are shown with significant ${ }^{* \star *} P<0.001$ in each subpanel. (C) Flow cytometry assays using indicated antibodies were performed after DMSO and ATRA-induced cell differentiation, respectively, with or without HMGA2 knockdown. Representative dot plots (measured at day 6 of treatment), and percentages of positive cells for indicated antigens are shown. The expression of CD11b, CD14 and CD15 was measured as described in 'Materials and methods' section. (D) Control or HMGA2-directed shRNA was combined with either $0.5 \%$ DMSO for HL-60 or $1 \mu$ M ATRA for NB4 for 6 days. Morphology of HL-60 and NB4 cells for each condition is described by MGG staining. Evidence of myeloid maturation contained an increase in cytoplasmic ruffling and a reduction in the nuclear-to-cytoplasmic ratio. Images were gained by an Olympus BX41 microscope, $\times 400$ magnification and Qcapture software. (E, F) The per cent of positive cells for indicated antigens is shown by flow cytometry. Error bars suggest the mean \pm s.d. of three replicates with statistical significance estimated by Student's $T$-test for each group. (Control vs shHMGA2, ${ }^{\star *} P<0.01$ for $\mathbf{E}$, ${ }^{\star} P<0.05$ for $\mathbf{F}$ ). 

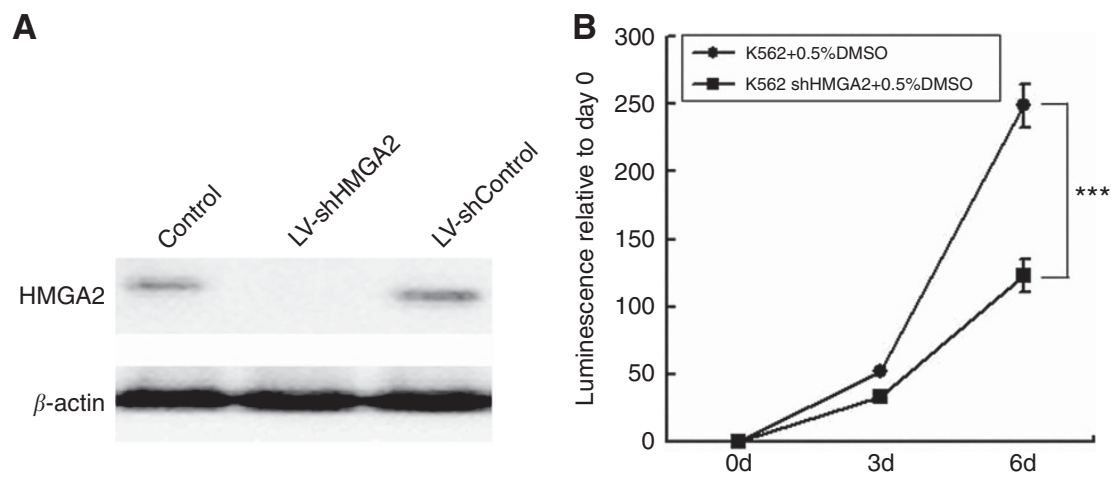

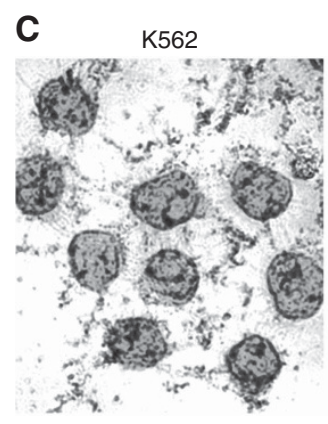

D

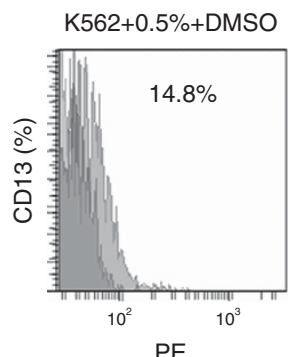

K562 shHMGA2+0.5\%+DMSO

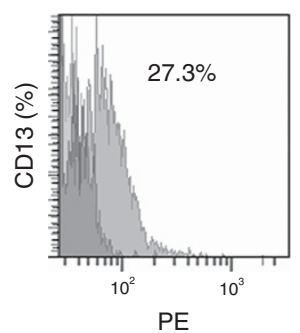

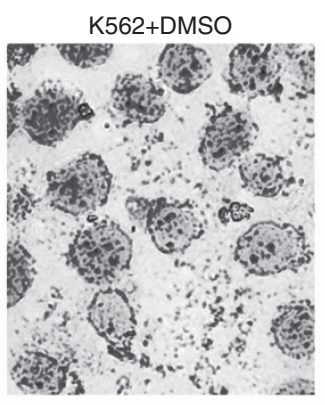

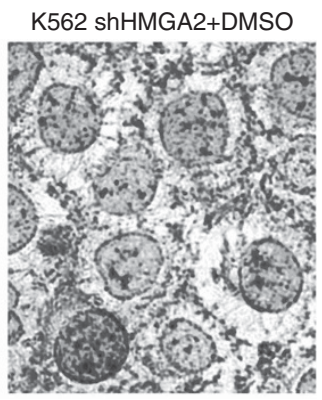

E

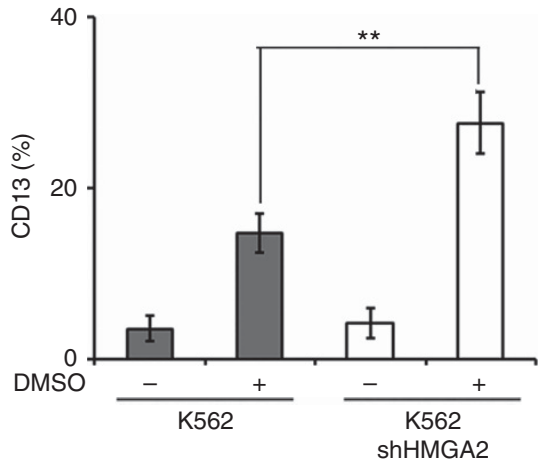

Figure 3. Chemical treatment increases the effect of genetic suppression of HMGA2 on cell viability and induces granulocytic maturation of K562 cells. (A) The result of western blot about HMGA2 protein in K562 cells, and $\beta$-actin is loading controls. (B) K562 cells with lentivirusShHMGA2 or lentivirus-ShControl were exposed to 0.5\% DMSO for K562 and viability was evaluated at 0, 3 and 6 days after treatment. Error bars suggest the mean \pm s.d. of three replicates with statistical significance estimated by Student's T-test for each compound at day 6 . All brackets are shown with significant ${ }^{\star \star \star} P<0.001$ in each subpanel. (C) HMGA2-directed shRNA or control was combined with either $0.5 \%$ DMSO for K562 for 6 days. Morphology of $\mathrm{K} 562$ cells for each condition is described by MGG staining. Evidence of granulocytic maturation consisted of a reduction in the nuclear-to-cytoplasmic ratio. Images were gained by an Olympus BX41 microscope, $\times 400$ magnification and Qcapture software. (D) Flow cytometry assays using indicated antibodies were performed after DMSO-induced K562 cell differentiation, with or without HMGA2 knockdown. Representative dot plots (measured at day 6 of treatment), and per cent of positive cells for indicated antigens are shown. The expression of CD13 was measured as described in 'Materials and methods' section. (E) The per cent of positive cells for indicated antigens is shown by flow cytometry. Error bars suggest the mean \pm s.d. of three replicates with statistical significance estimated by Student's T-test for each group. (Control vs shHMGA2, ${ }^{* *} P<0.01$ for $\left.\mathbf{E}\right)$.

(Figure 7A and B). The effects of HOXA9 low expression on cell differentiation were assessed. We found that an increase in CD14 or CD15 expression in AML cells was induced by down-regulation of HOXA9 (Figure 7C and D), which may contribute to induce the differentiation effect of HMGA2 knockdown.

\section{DISCUSSION}

Although man's understanding of the potential biological mechanisms in the pathogenesis of AML is developing all the time, poor survival rates intimate that new therapy tactics are still needed to 
A

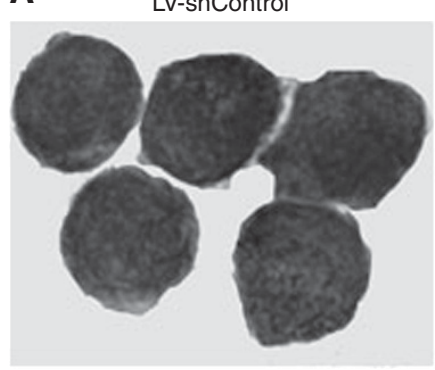

B

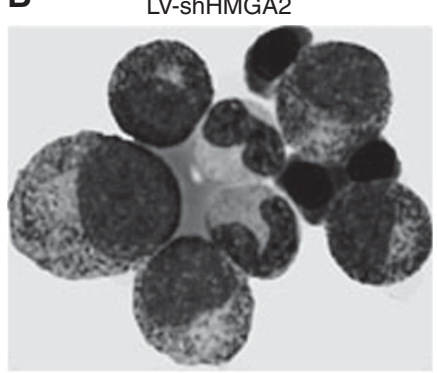

C

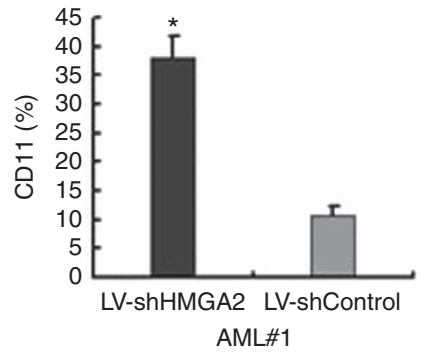

AML primary blasts

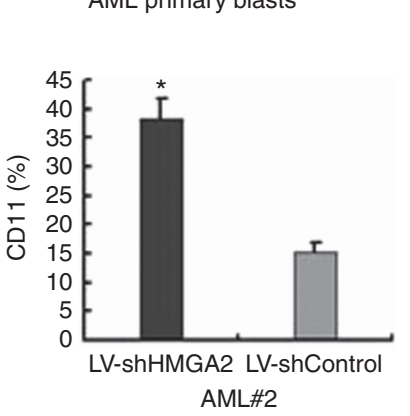

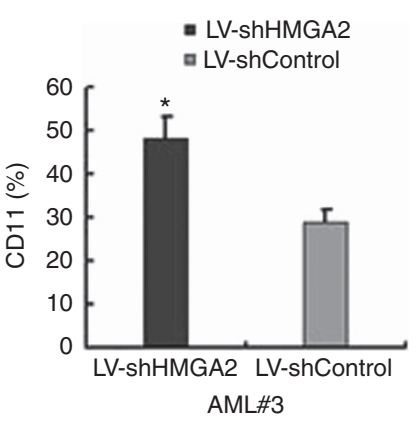

Figure 4. Transient transfection in AML primary cells with LV-shHMGA2 promotes cell differentiation. (A, B) Differentiation induced by IL-3 and GM-CSF and transient transfection (at day 2) with the shControl (A) or shHMGA2 (B) was assessed by cell morphology by MGG-stained cytocentrifuge slides after $6 \mathrm{~d}$ of differentiation. (C) Corresponding CD11b expression in primary blasts transfected with the shControl and primary blasts transfected with shHMGA2 were analysed by FACS, and the percentages of CD11b-positive cells are shown. Error bars suggest the mean \pm s.d. of three replicates with statistical significance estimated by Student's $T$-test for each group (Control vs shHMGA2, $\left.{ }^{\star} P<0.05\right)$.

A

\begin{tabular}{ccc}
\multicolumn{4}{c}{ HL-60 } \\
DMSO (days) \\
\hline Od & $3 d$ & $6 d$ \\
0.42 & $0 . .49$ & 0.17 \\
& & \\
\hline & & \\
\hline
\end{tabular}

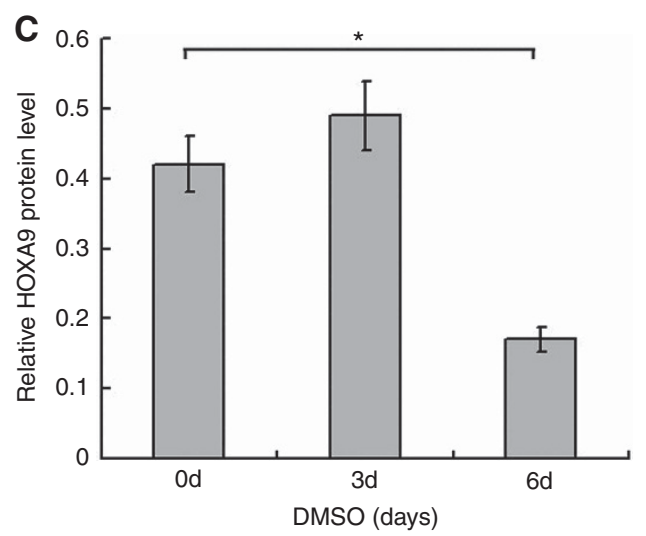

B
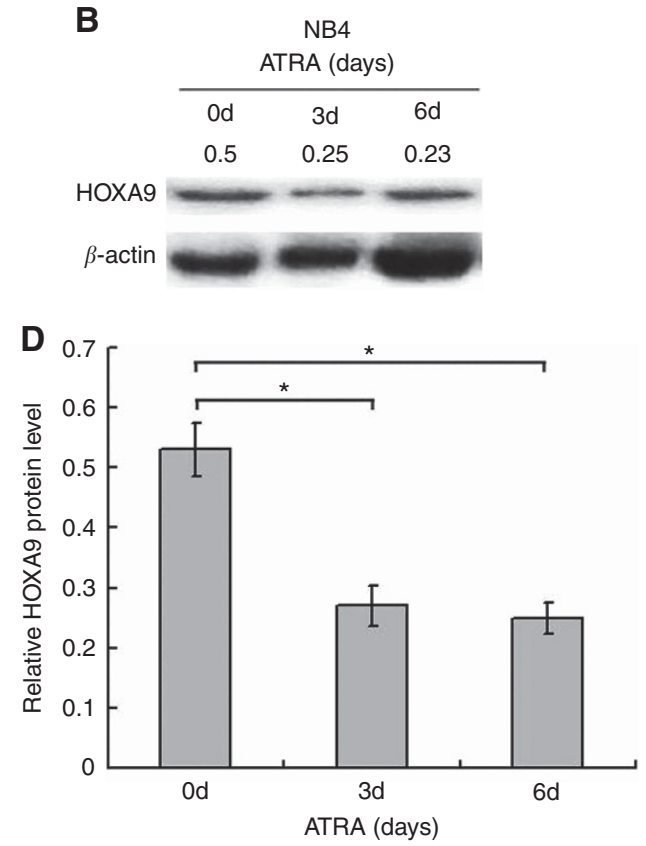

Figure 5. Endogenous HOXA9 expression during differentiation upon chemical treatment. (A, B) Endogenous HOXA9 protein expression of HL60 and NB4 cells during chemical treatment was analysed by western blot. $\beta$-actin was a loading control. (C, D) Densitometric analysis of HOXA9/ $\beta$-actin was shown by the numbers above the lanes. The western blots shown are from one experiment, which represents at least three independent experiments and cell lysates. Error bars suggest the mean \pm s.d. of three replicates with statistical significance evaluated using Student's $T$-test for each group ( $3 \mathrm{~d}$ or $6 \mathrm{~d}$ vs $0 \mathrm{~d}$, * $P<0.05$ ).

be studied. HMGA2 was recently confirmed as a novel target of AML in our laboratory (Tan et al, 2016), while there is little awareness of the role of HMGA2 in arrested differentiation of myeloid leukaemia. HMGA2 is expressed in CD34 + stem cells from healthy donors and blood from patients with myeloid leukaemia, while no expression was found in normal blood specimens. The overexpression of HMGA2 is related to the undifferentiated phenotype of the immature leukaemic cells (Andrieux et al, 2006; Meyer et al, 2007). Experimental data suggest a role for HMGA2 in malignant transformation, the inappropriate activation of the HMGA2 gene may be involved in myeloid cell transformation, suggesting that it could be the cause of 
A

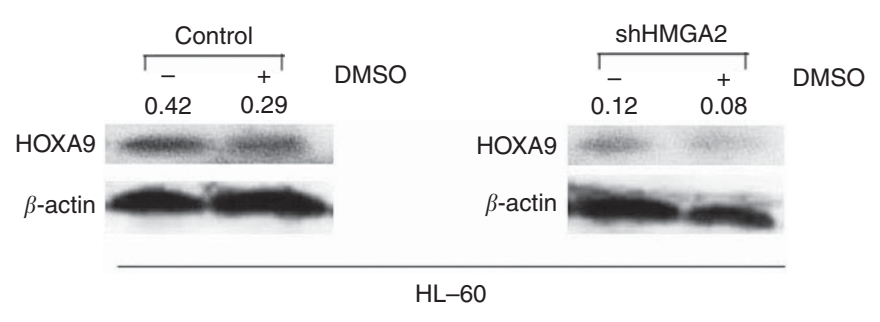

B

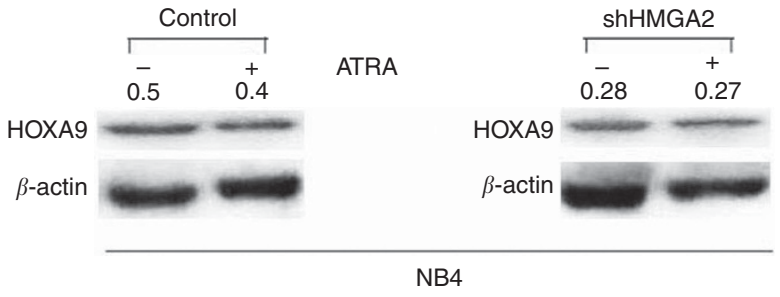

E

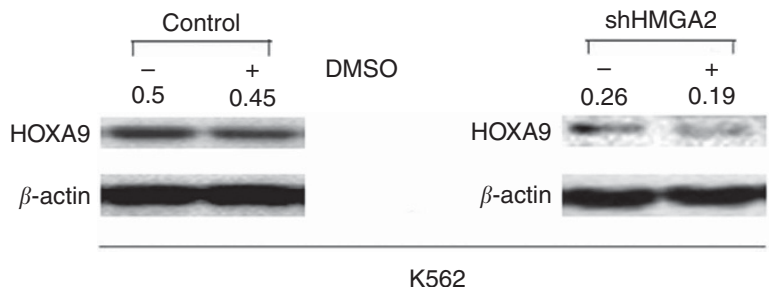

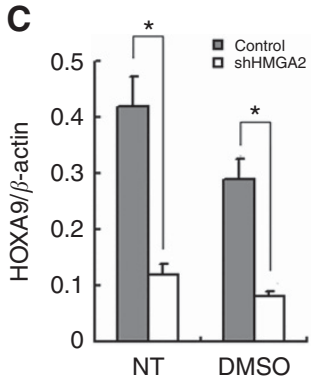

D

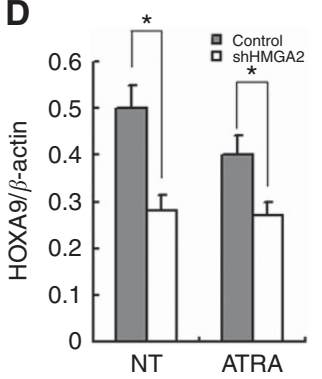

$\mathbf{F}$

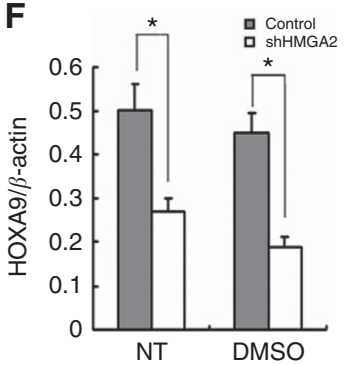

Figure 6. HOXA9 effectors are differentially modulated by HMGA2 in myeloid leukaemia. (A, B, E) Endogenous HOXA9 protein expression of HL-60, K562 and NB4 cells was analysed by western blot, which are treated or untreated with DMSO and ATRA (6 d), respectively. $\beta$-actin was loading control. (C, D, F) Densitometric analysis of HOXA9/ $\beta$-actin was shown by the numbers above the lanes. The intensity for each band was densitometrically quantified and the results of three independent experiments were shown in the histograms. Error bars suggest the mean $\pm \mathrm{s}$. $d$. of three replicates with statistical significance estimated by Student's $T$-test for each group (Control vs shHMGA2, ${ }^{\star} P<0.05$ ).

leukaemogenesis (Efanov et al, 2014). All this evidence points to a possible role for HMGA2 proteins in the development and differentiation of leukocytes and suggests that their deregulated expression may participate in the leukaemogenesis process in haematological lineages. HMGA2 is also aberrantly expressed in cancers, and its expression levels are inversely related with hepatocytic differentiation markers (Shell et al, 2007). The roles of HMGA2 in protecting tumour proliferation and inhibiting its differentiation were further highlighted by these findings. In view of all these studies, by inference, constant HMGA2 activation may prevent the blast cells from differentiating in human myeloid leukaemia. Under proper culture conditions, differentiation along the granulocytic and/or the monocytic lineage can be induced in AML cells, which have the bipotential granulo-monocytic essence (Bhatia et al, 1996; Brown et al, 2002). To investigate the role of HMGA2 in differentiation of myeloid leukaemia cells, we first assessed the expression of HMGA2 in the HL-60, NB4 myeloid cell lines upon chemical treatment and primary AML cells. We observed that HMGA2 mRNA has higher expression level in primary AML specimens, compared with normal samples (Figure 1A), and HMGA2 protein levels in primary AML specimens and myeloid leukaemia cell lines were also confirmed with high expression (Figure 1D). However, HMGA2 expression was gradually reduced with the increase of granulo-monocytic differentiation induced in NB4 and HL-60 upon chemical treatment (Figure $1 \mathrm{E}$ and $\mathrm{F}$ ). These results suggested that high expression of HMGA2 may have the inhibitory effect in differentiation and maturation of myeloid leukaemia cells, and the antidifferentiation role of HMGA2 in myeloid leukaemia cells is functionally correlated with its expression during granulomonocytic differentiation. So, we suspected that the expression of HMGA2 was enough to disturb the cellular differentiation in myeloid leukaemia. To elucidate this question, we checked whether the knockdown of HMGA2 can promote the differentiation of NB4, K562 and HL-60 leukaemic cells upon chemical treatment in transfection experiments. Under the given conditions, we can get a predominant mature monocytic cells from HL-60, and mature granulocytic cells from K562 cells (after DMSO treatment), whereas characteristic granulocytic differentiation can be seen in NB4 cells treated with retinoic acid (TARA;Bousquet et al, 2008). As shown in Figures 2A, B and 3B, by day 6, HMGA2 knockdown and exposure to these chemical treatments in leukaemic cells led to more lack of vitality than control with only DMSO or ATRA. The induction of monocytic differentiation maturation was shown by both morphology and increased expression of CD14 in HL-60 cells with the knockdown of HMGA2 (Figure 2C and D). We noticed that the maturation induced seemingly occurred in late stages of monocytic differentiation between the expression of CD11b and CD14 (Figure 2C and E).The gathering of CD11b and CD15 in granulocytic differentiation was obviously affected by shHMG2 
A

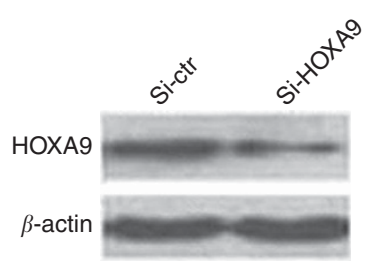

C

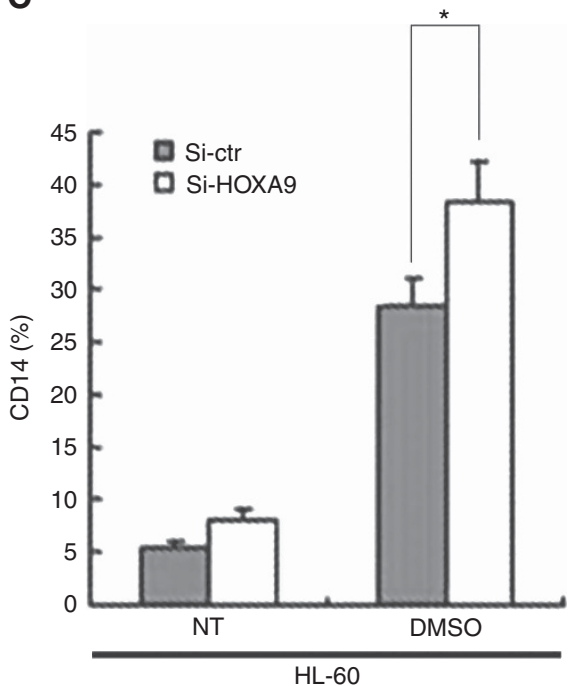

B

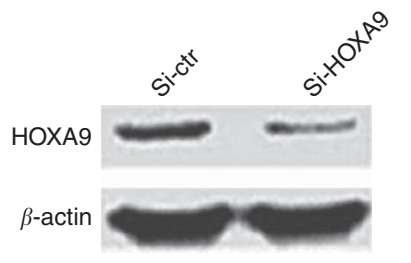

D

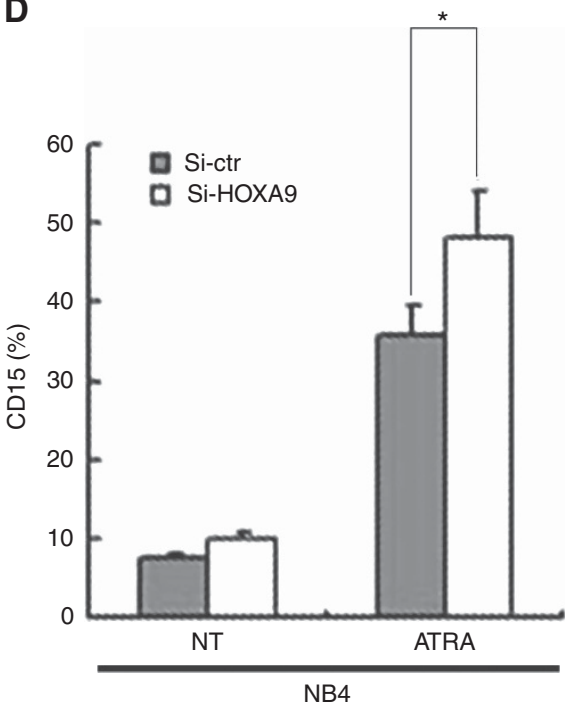

Figure 7. The role of HOXA9 expression during differentiation upon chemical treatment. (A, B) Expression of endogenous HOXA9 protein was analysed by western blot after electroporation of siRNA targeting human HOXA9 (siHOXA9, 600 nmol; ONTARGETplus SMARTpool, Dharmacon). There is no homology between the control RNA duplex (si-ctr $600 \mathrm{nmol}$; ON-TARGETplus Non-targeting pool, Dharmacon) for siRNA and any human gene sequences. For HOXA9 protein, the value is shown by a representative experiment of western blot. (C, D) The percentage of CD14 and CD15 expression cells was analysed, respectively, by FACS, after cells electroporated by siHOXA9 or si-ctr in the NB4 and HL-60 cell lines, untreated or treated with DMSO and ATRA $(72 \mathrm{~h})$, respectively. Error bars suggest the mean $\pm \mathrm{s} . \mathrm{d}$. of three replicates with statistical significance estimated by Student's $T$-test for each group (Control vs shHMGA2, ${ }^{*}<<0.05$ ).

stable transfection in NB4 cells upon ATRA treatment (Figure 2C and F). While the number of granulocytic maturation cells significantly increased in K562 cells with knockdown of HMGA2 (Figure 3C) after co-incubation with $0.5 \%$ DMSO for 6 days, the expression of CD13 was also significantly higher than that in K562 cells (Figure 3D and E). The maturation induced was confirmed by morphological analysis, and HL-60, K562 and NB4 morphological analyses showed a strengthened level of cytoplasmic ruffling and nuclear condensation in the conditions combining knockdown of HMGA2 and chemical treatment of DMSO or ATRA. The combination of HMGA2-directed shRNAs and the chemical treatment of DMSO or ATRA led to a higher level of myeloid differentiation than control with DMSO or ATRA consistent with the morphological findings (Figures $2 \mathrm{D}$ and $3 \mathrm{C}$ ). HMGA2 inhibition promotes greater differentiation than control, suggesting that HMGA2 knockdown is likely contributing to the differentiation induced by chemicals in myeloid leukaemia. HMGA2 knockdown in NB4, HL-60 and K562 cells induced cell morphology with more maturation. Together, these results strongly indicated that shHMGA2 induces monocyticgranulocytic differentiation of myeloid leukaemia cells, which was further proved in primary cells, and we found that HMGA2 knockdown significantly increased CD11b upregulation in AML primary cells (Figure 4C). However, the precise mechanism by which HMGA2 inhibition results in this enhanced differentiation is not yet established, some data indicated that HOXA9 is critical to normal myelopoiesis and its expression must be downregulated to permit normal differentiation (Sauvageau et al, 1994). More and more evidence demonstrated that HOXA9 also intervened orientation stages of haemopoietic stem cells and the late differentiation, its overexpression keeps the self-renewal capacity of leukaemic stem cells and their differentiation was also blocked, which contributed to leukaemogenesis (Thorsteinsdottir et al, 2002; Faber et al, 2009). Furthermore, HOXA9 is upregulated during transiting in chronic myeloid leukaemia patients to blastphase disease, and HOXA9 expression itself is an independent risk factor in children with leukaemia (Tedeschi and Zalazar, 2006; Adamaki et al, 2015). Persistent expression of HOXA9 results in myeloid differentiation arrest, and inactivation of HOXA9 that promotes the terminal neutrophil differentiation in primary murine bone marrow mononuclear cells generates factor-dependent GMP cell lines (Kroon et al, 1998). Next, the effect of ATRA or DMSO on HOXA9 protein expression of myeloid leukaemia cells was evaluated. In our study, we found that HOXA9 expression in HL-60 and NB4 was obviously affected by DMSO and ATRA treatment. HOXA9 expression was reduced during the process of granulo-monocytic nature of AML cells (Figure 5A and B), which was consistent with the change of HMGA2 in the differentiation of AML cells (Figure 1E). Interestingly, HOXA9 mRNA relative expression levels were significantly upregulated in 10 new AML patients, compared with the controls (Figure 1B). Spearmann correlation coefficients showed that HOXA9 expression was related with expression of HMGA2 in 7 identical novo AML patients $(r=0.883, P<0.001$; Figure $1 \mathrm{C})$. The expression of HOXA9 may be regulated by HMGA2, so the relationship between HOXA9 and HMGA2 was further investigated. We found that HMGA2 knockdown in HL-60, K562 and NB4 significantly inhibited HOXA9 expression (Figure 6A, B and E), and HOXA9 expression 
was also significantly decreased in HL-60, K562 and NB4 with HMGA2 knockdown and upon DMSO or ATRA treatment (Figure 6C, D and F). These results suggest that HOXA9-positive regulation is mediated, at least in part, by HMGA2. Finally, to estimate whether HOXA9 is considered as a target participating in inducing the differentiation effect of shHMGA2 in AML, the effects of HOXA9 knockdown on cell differentiation were evaluated. We found that HOXA9 down-regulation (Figure 7A and $\mathrm{B})$, respectively, induced an increase in the expression of CD14 and CD15 in HL-60 and NB4 cells (Figure 7C and D), indicating that it helps the pro-differentiating function of shHMGA2. These data suggest that HMGA2 and HOXA9 may be considered as target genes participating in arrested differentiation of myeloid cells. Moreover, HOXA9 down-regulation in protein level by ATRA in NB4 cells or DMSO in HL-60 may be, in part, mediated by HMGA2. In conclusion, we have found that shHMGA2 promotes myeloid differentiation in myeloid leukaemia, and have experimentally validated and identified HOXA9 as a new HMGA2regulating target in cell differentiation of myeloid. It has been reported that HMGA2/TET1/HOXA9 signalling pathway in the epigenetic regulation of human breast cancer may play an important role of targeting methylation in specific subpopulations, and the signalling pathway may regulate breast cancer metastasis and growth, and contains a prognostic signature for patient survival (Sun et al, 2013). However, the pathological function of TET1 emphasises the importance of cell context, so the mechanisms of interaction between HMGA2 and HOXA9 in cell differentiation of myeloid leukaemia will require further investigation. HMGA2 does not show direct transcriptional activation capacity, but modulates gene expression by changing the DNA conformation and binding to AT-rich regions in the DNA and/or direct interaction with several transcription factors. By this means, HMGA2 proteins take part in multifarious nuclear processes ranging from chromosome and chromatin dynamics to serving as architectural transcription factors that modulate the expression of numerous genes (Cleynen and Van de Ven, 2008), so we speculate the feasibility with which HMGA2 might regulate HOXA9 expression by direct combination with the HOXA9 promoter or by changing its chromatin structure or regulating HOXA9 expression indirectly via other mediators. These problems about the association of HMGA2 with HOXA9 in cell differenation of myeloid leukaemia will be our further study. Some studies reported that the interaction with MLL or TET1 may regulate HOXA9 expression. If the HOXA9 and HMGA2 are linked during myeloid differentiation through protein-protein interactions, it is possible that HMGA2 forms a complex with HOXA9 and co-regulates common downstream target genes in myeloid differentiation. If none of HMGA2-binding sites in myeloid leukaemia cells are near to the genomic loci of HOXA9 gene, it is possible that HMGA2 indirectly modulates HOXA9 expression via other mediators, such as a signalling cascade whereby HMGA2 inhibits myeloid leukaemia cell differentiation in part through inhibition of other protein-mediated HOXA9 gene expression which will also be studied in the further project. As HMGA2 and HOXA9 have all been implicated in blockage of differentiation in myeloid leukaemia, our findings offer new information on the pathogenesis of myeloid leukaemia. Perturbation of the HMGA2-HOXA9 pathway may have a therapeutic value in myeloid leukaemia.

\section{ACKNOWLEDGEMENTS}

This work was supported by the National Natural Science Foundation of China (No. 81100379), Guangzhou Medical and Health Technology Program (20171A011289), and the Medical
Scientific Research Foundation of Guangdong Province, China (No. A2014292).

\section{CONFLICT OF INTEREST}

The authors declare no conflict of interest.

\section{REFERENCES}

Adamaki M, Lambrou GI, Athanasiadou A, Vlahopoulos S, Papavassiliou AG, Moschovi M (2015) HOXA9 and MEIS1 gene overexpression in the diagnosis of childhood acute leukemias: Significant correlation with relapse and overall survival. Leuk Res 39: 874-882.

Andrieux J, Bilhou-Nabera C, Lippert E, Le Bousse-Kerdiles MC, Dupriez B, Grardel N, Pierre-Louis O, Desterke C, Praloran V, Laï JL, Demory JL (2006) Expression of HMGA2 in PB leukocytes and purified CD34 + cells from controls and patients withMyelofibrosis and myeloid metaplasia. Leuk Lymphoma 47: 1956-1959.

Bennett JM, Catovsky D, Daniel MT, Flandrin G, Galton DA, Gralnick HR, Sultan C (1985) Proposed revised criteria for the classification of acute myeloid leukemia. A report of the French-American-British Cooperative Group. Ann Intern Med 103: 620-625.

Bhatia M, Kirkland JB, Meckling-Gill KA (1996) Overexpression of poly(ADP-ribose) polymerase promotes cell cycle arrest and inhibits neutrophilic differentiation of NB4 acute promyelocytic leukemia cells. Cell Growth Differ 7: 91-100.

Bousquet M, Quelen C, Rosati R, Mansat-De Mas V, La Starza R, Bastard C, Lippert E, Talmant P, Lafage-Pochitaloff M, Leroux D, Gervais C, Viguié F, Lai JL, Terre C, Beverlo B, Sambani C, Hagemeijer A, Marynen P, Delsol G, Dastugue N, Mecucci C, Brousset P (2008) Myeloid cell differentiation arrest by miR-125b-1 in myelodysplastic syndrome and acutemyeloid leukemia with the $\mathrm{t}(2 ; 11)(\mathrm{p} 21 ; \mathrm{q} 23)$ translocation. J Exp Med 205: 2499-2506.

Brown G, Drayson MT, Durham J, Toellner KM, Hughes PJ, Choudhry MA, Taylor DR, Bird R, Michell RH (2002) HL60 cells halted in G1 or S phase differentiate normally. Exp Cell Res 281: 28-38.

Cleynen I, Van de Ven WJ (2008) The HMGA proteins: a myriad of functions. Int J Oncol 32: 289-305.

Efanov A, Zanesi N, Coppola V, Nuovo G, Bolon B, Wernicle-Jameson D, Lagana A, Hansjuerg A, Pichiorri F, Croce CM (2014) Human HMGA2 protein overexpressed in mice induces precursor T-cell lymphoblastic leukemia. Blood Cancer J 4: e227.

Eppinger TM, Buck J, Hämmerling U (1993) Growth control or terminal differentiation: endogenous production and differential activities of vitamin A metabolites in HL-60 cells. J Exp Med 178: 1995-2005.

Faber J, Krivtsov AV, Stubbs MC, Wright R, Davis TN, van den HeuvelEibrink M, Zwaan CM, Kung AL, Armstrong SA (2009) HOXA9 is required for survival in human MLL-rearranged acute leukemias. Blood 113: 2375-2385.

Gao Z, Golob J, Tanavde VM, Civin CI, Hawley RG, Cheng L (2001) High levels of transgene expression following transduction of long-term NOD/ SCID-repopulatinghuman cells with a modified lentiviral vector. Stem Cells 19: 247-259.

Golub TR, Slonim DK, Tamayo P, Huard C, Gaasenbeek M, Mesirov JP, Coller H, Loh ML, Downing JR, Caligiuri MA, Bloomfield CD, Lander ES (1999) Molecular classification of cancer: class discovery and class prediction by gene expression monitoring. Science 286: 531-537.

Kroon E, Krosl J, Thorsteinsdottir U, Baban S, Buchberg AM, Sauvageau G (1998) Hoxa9 transforms primary bone marrow cells through specific collaboration with Meis1a but notPbx1b. EMBO J 17: 3714-3725.

McCulloch EA (1983) Stem cells in normal and leukemic hemopoiesis (Henry Stratton Lecture, 1982). Blood 62: 1-13.

Meyer B, Krisponeit D, Junghanss C, Murua Escobar H, Bullerdiek J (2007) Quantitative expression analysis in peripheral blood of patients with chronic myeloid leukaemia:correlation between HMGA2 expression and white blood cell count. Leuk Lymphoma 48: 2008-2013.

Odero MD, Grand FH, Iqbal S, Ross F, Roman JP, Vizmanos JL, Andrieux J, Laï JL, Calasanz MJ, Cross NC (2005) Disruption and aberrant expression of HMGA2 as a consequence of diverse chromosomal translocations in myeloid malignancies. Leukemia 19: 245-252. 
Sauvageau G, Lansdorp PM, Eaves CJ, Hogge DE, Dragowska WH, Reid DS, Largman C, Lawrence HJ, Humphries RK (1994) Differential expression of homeobox genes in functionally distinct CD34 + subpopulations ofhuman bone marrow cells. Proc Natl Acad Sci USA 91: 12223-12227.

Shell S, Park SM, Radjabi AR, Schickel R, Kistner EO, Jewell DA, Feig C, Lengyel E, Peter ME (2007) Let-7 expression defines two differentiation stages of cancer. Proc Natl Acad Sci USA 104: 11400-11405.

Stone RM (2009) The difficult problem of acute myeloid leukemia in the older adult. CA Cancer J Clin 52: 363-371.

Sun M, Song CX, Huang H, Frankenberger CA, Sankarasharma D, Gomes S, Chen P, Chen J, Chada KK, He C, Rosner MR (2013) HMGA2/TET1/ HOXA9 signaling pathway regulates breast cancer growth and metastasis. Proc Natl Acad Sci USA 110: 9920-9925.

Tan L, Wei X, Zheng L, Zeng J, Liu H, Yang S, Tan H (2016) Amplified HMGA2 promotes cell growth by regulating Akt pathway in AML J Cancer Res Clin Oncol 142: 389-399.

Tedeschi FA, Zalazar FE (2006) HOXA9 gene expression in the chronic myeloid leukemia progression. Leuk Res 30: 1453-1456.
Thorsteinsdottir U, Kroon E, Jerome L, Blasi F, Sauvageau G (2001) Defining roles for HOX and MEIS1 genes in induction of acute myeloid leukemia. Mol Cell Biol 21: 224-234.

Thorsteinsdottir U, Mamo A, Kroon E, Jerome L, Bijl J, Lawrence HJ, Humphries K, Sauvageau G (2002) Overexpression of the myeloid leukemia-associated Hoxa9 gene in bone marrow cells inducesstem cell expansion. Blood 99: 121-129.

Wang ZY, Chen Z (2008) Acute promyelocytic leukemia: from highly fatal to highly curable. Blood 111: 2505-2515.

Yam LT, Li CY, Crosby WH (1971) Cytochemical identification of monocytes and granulocytes. Am J Clin Pathol 55: 283-290.

Zhao P, Tan L, Ruan J, Wei XP, Zheng Y, Zheng LX, Tan H (2015) Aberrant expression of HOXA5 and HOXA9 in AML. Asian Pac J Cancer Prev 16(9): 3941-3944.

This work is published under the standard license to publish agreement. After 12 months the work will become freely available and the license terms will switch to a Creative Commons AttributionNonCommercial-Share Alike 4.0 Unported License. 